\title{
An evolutionary consequence of dosage compensation on Drosophila melanogaster female X-chromatin structure?
}

\author{
Yu Zhang ${ }^{*}$, Brian Oliver
}

\begin{abstract}
Background: $X$ chromosomes are subject to dosage compensation in Drosophila males. Dosage compensation requires cis sequence features of the $X$ chromosome that are present in both sexes by definition and trans acting factors that target chromatin modifying machinery to the $X$ specifically in males. The evolution of this system could result in neutral $X$ chromatin changes that will be apparent in females.

Results: We find that the general chromatin structure of female $X$ chromosomes is distinct from autosomes. Additionally, specific histone marks associated with dosage compensation and active chromatin marks on the male $X$ chromosome are also enriched on the $X$ chromosomes of females, albeit to a lesser degree.

Conclusions: Our data indicate that $X$ chromatin structure is fundamentally different from autosome structure in both sexes. We suggest that the differences between the $X$ chromosomes and autosomes in females are a consequence of mechanisms that have evolved to ensure sufficient $X$ chromosome expression in the soma of males.
\end{abstract}

\section{Background}

Drosophila X chromosomes show peculiar features in both gene expression [1] and gene evolution [2]. One of the most striking consequences of $\mathrm{X}$ chromosome hemizygosity in males, is dosage compensation, a process which brings $\mathrm{X}$ chromosome and autosome expression into balance [3-5]. Dosage compensation was probably acquired gradually in the course of sex chromosome evolution, as sex chromosomes are thought to arise by divergence of an ancestral autosome pair [6]. Gene loss from the $\mathrm{Y}$ chromosome creates an increasingly aneuploid condition in males and is thought to be the driving force in the evolution of global X-chromosome dosage compensation. In the absence of dosage compensation genomic imbalance results in male lethality.

It has long been known that selective pressures applied to just one of the sexes can effect change in the other [7]. For example, the coloration of certain birds or the nipples of mammals are advantageous to one of the

\footnotetext{
* Correspondence: yuzhang@niddk.nih.gov; oliver@helix.nih.gov Laboratory of Cellular and Developmental Biology, National Institute of Diabetes and Digestive and Kidney Diseases, National Institutes of Health, Bethesda MD 20892-8028 USA
}

sexes and are likely to be present as an evolutionary side-effect in the other. X chromosome dosage compensation might also show evidence of this type of sexual selection. X chromosome dosage compensation requires both cis and trans components [1]. Cis changes resulting from selection of the compensation system in males will also be present in females, and might alter the character of the X chromosome in females as a secondary consequence [8]. Indeed, we have previously noted a slight over-expression of both male and female $\mathrm{X}$ chromosomes relative to autosomes $[4,9]$, which suggests that the $\mathrm{X}$ chromosome is inherently more active than autosomes. We have therefore examined the structure of $\mathrm{X}$ chromatin in females in detail.

Expression patterns and especially $\mathrm{X}$ chromosome dosage compensation are mediated by chromatin modification $[1,10,11]$. Histones are nucleosome subunits required for packing DNA into the confines of the nucleus. It has long been know that chromatin structure changes are associated with transcription [12]. For example, when chromatin is physically sheared to small fragments by sonication or enzyme digestion, shearingbias is associated with different chromatin structures 
across the genome [13-15]. Histones are also modified on $\mathrm{N}$-terminal tail residues to generate an expanding repertoire of histone modifications that are important modulators of transcription [16,17]. It has become increasingly clear that specific types of modification are associated with particular transcriptional outcomes. For example, acetylation events are broadly associated with transcriptional activation, while methylation events can have either activating or repressing roles.

One of the best studied histone modifications is the acetylation of Histone 4 on Lysine 16 (H4K16ac). In organisms from yeast to humans, H4K16ac is broadly associated with active genes, and the Histone Acetyl Transferase (HAT) that writes the modification is required for viability [18-21]. In Drosophila, H4K16ac is highly enriched on the $\mathrm{X}$ chromosomes of males $[22,23]$, and the responsible HAT, Males Absent on First (Mof), is required for male viability [21]. While Mof is associated with some genes in both males and females [24], Mof is greatly enriched on the male $\mathrm{X}$ chromosome due to targeting by the male-specific-lethal (MSL) complex. MSL is composed of proteins (Mle, Msl1, Msl2, Msl3, and Mof) and two non-coding RNAs encoded on the X (RoX1 and RoX2) [1]. It is thought that the greatly increased H4K16ac levels act to increase $\mathrm{X}$ chromosome expression in males, although it is also possible that $\mathrm{X}$ chromosome enrichment depletes autosomes of H4K16ac $[1,25]$. In either model, X chromosome and autosome expression are equilibrated to restore transcription balance.

Another chromatin modifying enzyme, Jil1, is also enriched on the X chromosome of males [26-28]. This kinase mediates phosphorylation of Histone 3 at serine 10 (H3S10ph). Jil1 is required for full dosage compensation and associates with the MSL complex $[27,29]$. H3S10ph is implicated in both chromosome condensation during mitosis and transcriptional activation during interphase, suggesting that Jil1 has more general roles in addition to dosage compensation. Another mark associated with active transcription, dimethylation of histone $\mathrm{H} 3$ at lysine 4 (H3K4me2) [17] is general, and thus likely to be MSL complex independent.

We have performed chromatin-shearing experiments showing that X chromatin differs from autosomal chromatin in both males and females. Additionally, the histone marks associated with $\mathrm{X}$ chromosome dosage compensation in males are modestly enriched on female $\mathrm{X}$ chromosomes. These data indicate that $\mathrm{X}$ chromatin is distinct even in the absence of dosage compensation. We suggest that the pattern in females is a tolerated neutral side-effect of the evolution of $\mathrm{X}$ chromosome dosage compensation in males.

\section{Results}

Global analysis of chromatin structure in females and males

We took advantage of differential shearing to probe chromatin structure by deep DNA sequencing (DNASeq) [30]. Specifically, we sheared cross-linked chromatin, size selected for short (200 bp) fragments, performed deep sequencing, and aligned the DNA reads to the reference genome. Because these sequencing reactions generated reads from the ends of size-selected fragments, increased mapped read density occurs in regions of preferential shearing.

We constructed libraries of DNA from sheared chromatin from female and male adult flies and obtained about 3 million uniquely mapping 35 bp reads for each sex. We then parsed the mapped reads by chromosome arms. The $\mathrm{X}$ chromosome and each arm of the $2^{\text {nd }}$ and $3^{\text {rd }}$ chromosomes bear about $20 \%$ of the genome, while the $4^{\text {th }}$ chromosome bears $<1 \%$. The average coverage of the different chromosomes (depth of sequence coverage at each base in non-overlapping $1 \mathrm{~kb}$ windows normalized by total sequenced base pairs) was similar (average coverage from 6.81-8.49 for chromosome arms in males and 5.91-7.23 in females) except the $\mathrm{X}$ chromosome in males (average coverage 3.71 ) and the $4^{\text {th }}$ chromosome in both males and females (average coverage 1.54 and 1.68) (Figure 1A, B). The average coverage of the male $\mathrm{X}$ chromosome was $48 \%$ of the autosome coverage, which is consistent with the male karyotype. Permutation testing of chromosome arm sequence coverage relative to genome-wide coverage showed that the $4^{\text {th }}$ chromosome had unusually low read density (Figure $1 \mathrm{C}$, D). The under-representation of DNA sequence coverage on the heterochromatin-rich, gene poor, the $4^{\text {th }}$ chromosome [31] suggests that this chromosome was resistant to shearing. Thus, the $4^{\text {th }}$ chromosome data suggests that lower sequence coverage is associated with a more "closed" chromatin state in this largely heterochromatin chromosome.

We were interested in shearing at gene models, as chromatin alternations associated with transcription, and thus dosage compensation, should be evident at those sites. To summarize the gene model read density patterns genome-wide, we calculated read coverage at multiple gene model features. We clearly observed higher sequence coverage at exonic regions relative to intronic regions and elevated coverage flanking transcription start and termination sites on $\mathrm{X}$ chromosomes and autosomes (Figure 2A-H). We suggest that these signatures are due to association of different proteincomplex types during transcription. Thus, like DNase hypersensitivity [32], mechanically sheared chromatin 

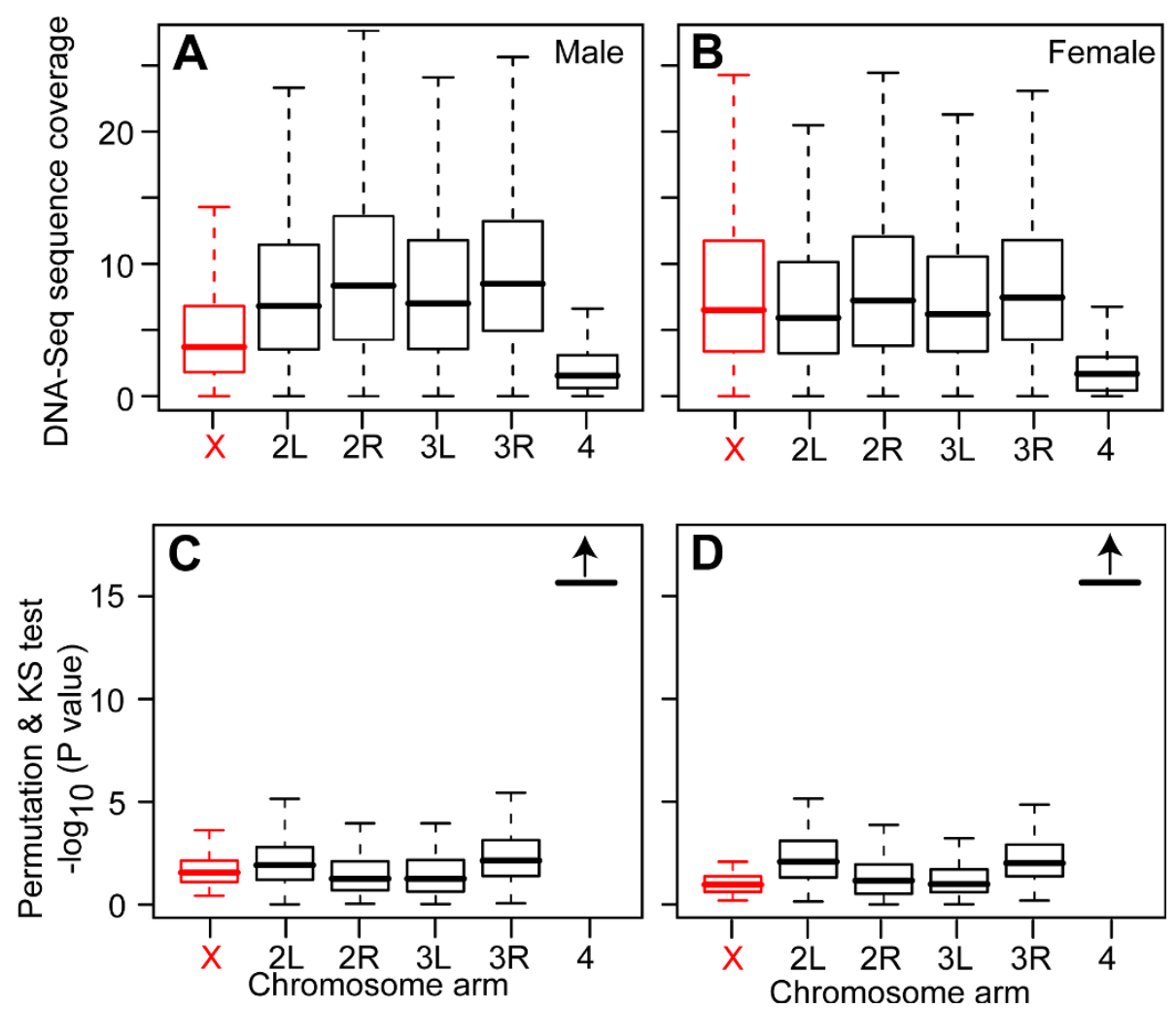

Figure 1 Sheared chromatin DNA-Seq coverage in male and female adult flies. (A-B) Box plots of the distributions of average DNA-Seq sequence coverage (in non-overlapping $1 \mathrm{~kb}$ windows) in male (A) and female (B) adult flies separated by chromosome arms ( $X$ chromosome in red). 25th to 75th percentiles (boxes), medians (lines in boxes), and ranges (whiskers, 1.5 times the interquartile range extended from both end of the box ) are indicated for each chromosome. Significance of the chromosome arm distributions relative to the whole genome data set as determined by permutation sampling and KS test (C, D).

breaks preferentially at some sites to provide information about chromatin structure genome-wide.

We then compared the coverage profiles of X chromosome and autosome gene model features inter se to determine if the structure of the $\mathrm{X}$ chromosome differed from the autosomes. Indeed, the average coverage for $\mathrm{X}$ chromosome genes was clearly higher than autosome arm genes at exons, transcription start sites, and transcription termination sites in both males and females (Figure 2A-H). The $4^{\text {th }}$ chromosome always showed greatly reduced sequence coverage. Permutation testing clearly suggests that the $\mathrm{X}$ chromosome shearing at exons, transcription start sites, and termination sites is significantly greater than the pattern genome-wide in both males (not shown) and females (Figure 2I-L). These data indicate that $\mathrm{X}$ chromosome genes are susceptible to shearing, probably as result of a more open structure.

\section{Distribution of histone marks on female and male chromosomes}

If cis features have evolved on the $\mathrm{X}$ chromosome to facilitate dosage compensation in conjunction with the trans-acting MSL complex, then the $\mathrm{X}$ chromosome in females might show a "shadow" profile resembling the male X chromosome. We therefore more specifically probed for the histone modifications that are associated with $\mathrm{X}$ chromosome dosage compensation. To localize histone marks in the genome, we carried out chromatin immunoprecipitation coupled with microarray hybridization (ChIP-chip) to FlyGEM arrays on sex-sorted adult flies. The DNA from ChIP and input chromatin were hybridized to the same slides, normalized, and averaged to generate a ratio for each gene (see methods). In agreement with the DNA-Seq coverage data, the intensity of ChIP input X chromosome DNA was significantly higher than the input for the autosomes in females and was significantly lower for the $4^{\text {th }}$ chromosome (Bonferroni corrected KS test, Figure 3A). Thus, the input data also suggest that $\mathrm{X}$ chromatin is more open. ChIP ratios (ChIP enriched DNA/input) eliminate the effect of chromatin on the input channel when testing for histone modification enrichment.

As expected, we found a strong enrichment for $\mathrm{H} 4 \mathrm{~K} 16 \mathrm{ac}$ on the male $\mathrm{X}$ chromosome relative to male 


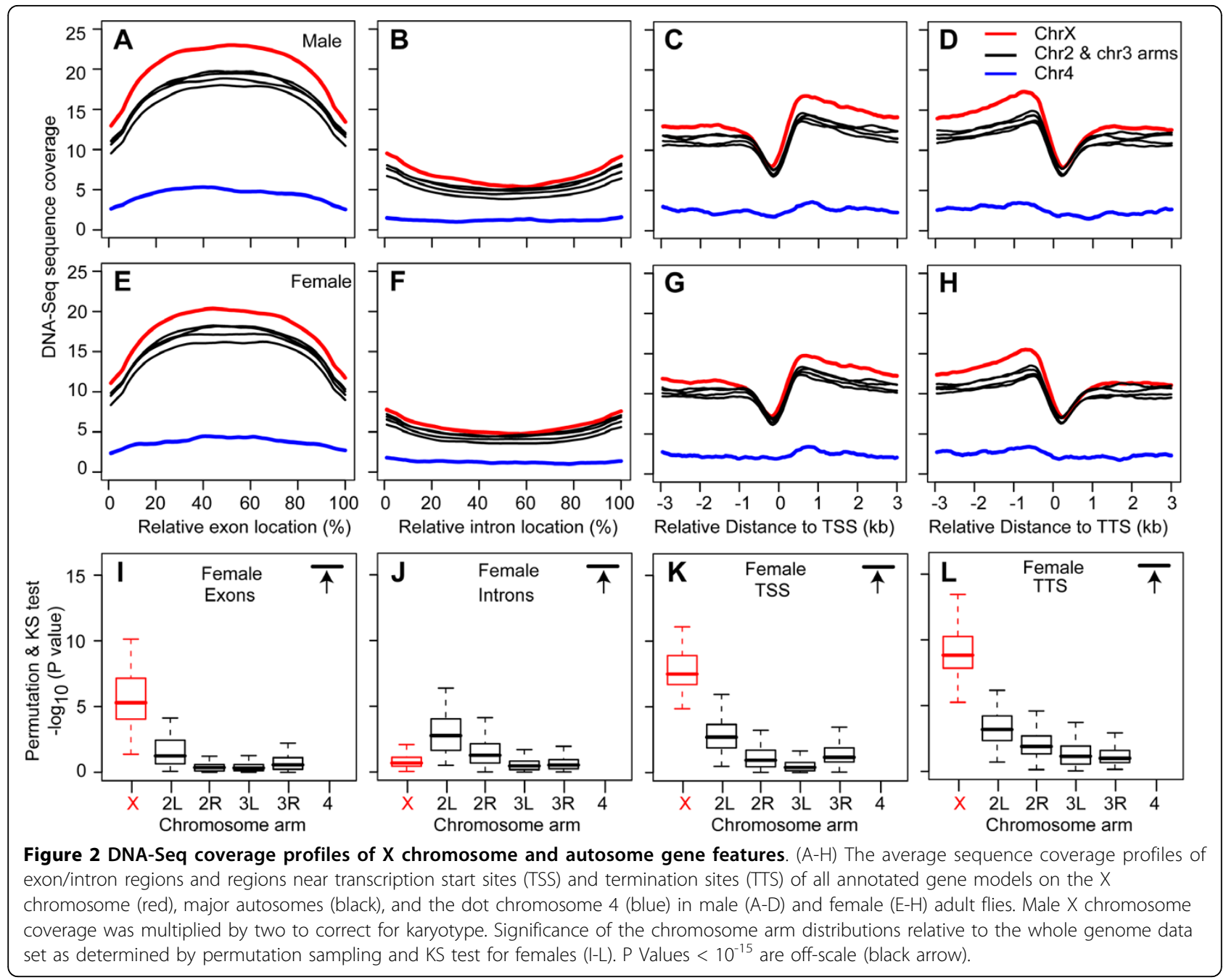

autosomes (Figure 3B, E). The average H4K16ac ChIP ratio (ChIP enriched DNA/input) on male $\mathrm{X}$ chromosome genes was 1.39 fold higher than the value of all autosome gene. These data show that we are able to easily score the high levels of H4K16ac present on the male $\mathrm{X}$ chromosomes in adult flies. Interestingly, we observed a modest (1.06 fold) but significant enrichment for $\mathrm{H} 4 \mathrm{~K} 16 \mathrm{ac}$ on female $\mathrm{X}$ chromosomes relative to all the autosomes (Figure 3B, H). In both males and females, the ratio of H4K16ac ChIP/input was similar for all major autosome arms (2L, 2R, 3L, 3R) indicating that there are no differences in H4K16ac among the major autosome arms within either sex. These data indicate that not only is $\mathrm{X}$ chromatin different from autosomal chromatin in females, but that the important dosage compensation mark H4K16ac is generally enriched on X chromosomes.

We observed a modest, but significant 1.09 fold enrichment for the H3S10ph mark on the male X chromosome relative to the autosomes (Figure $3 \mathrm{C}, \mathrm{F}$ ). We also observed a 1.02 fold enrichment of H3S10ph on the female $\mathrm{X}$ chromosome, but this difference was only modestly significant (Figure 3C, I). As observed for the other two histone marks, we found a significant enrichment for H3K4me2 on the $\mathrm{X}$ chromosome in males (Figure 3D, G). But again, we also found significant enrichment for H3K4me2 on the female X chromosome (Figure 3D, J). Thus, the patterns of histone mark accumulation on the male $\mathrm{X}$ chromosome appear to be shadowed by similar distributions on the female $\mathrm{X}$ chromosome.

Distributions of histone marks correlate with each other and active genes

If the female $\mathrm{X}$ chromosome shadows the pattern observed on the male $\mathrm{X}$ because of underlying changes in $\mathrm{X}$ chromosome sequence, then the histone marks should also correlate at the gene level. To determine if the same genes show similar histone modifications on both the male and female $\mathrm{X}$ chromosome, we directly examined correlations by using the rank order of 


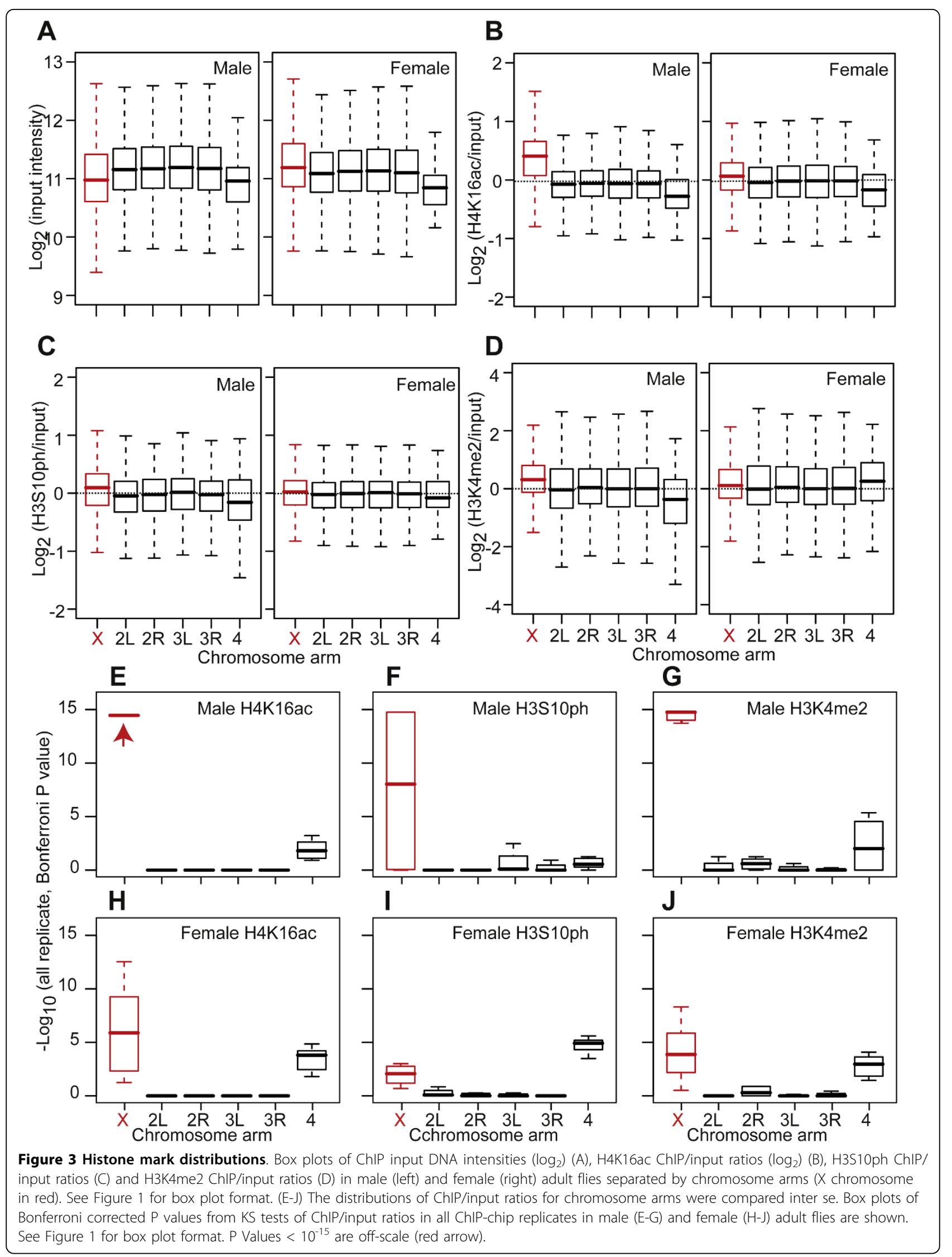


individual histone marks to normalize the results of the three distinct ChIP data sets and then subjected the rank order data to cluster analysis (Figure 4). To highlight associations on the autosomes, we clustered the $\mathrm{X}$ chromosomes and autosomes separately. We observed a strong positive correlation between H4K16ac and the other two histone marks on both $\mathrm{X}$ chromosomes and autosomes. For example, Spearman's Rank Correlation indicates that H4K16ac on male X chromosomes was positively correlated with H3K4me2 (Spearman's Rho = 0.64 ) and H3S10ph (Spearman's Rho $=0.4$ ). On the autosomes, H4K16ac enrichment was positively correlated with female H4K16ac (Spearman's Rho = 0.69) and with other two histone modifications (Spearman's Rho ranged from 0.40-0.49). More importantly, there was a good rank correlation between male and female X chromosomes for H4K16ac (Spearman's Rho = 0.46), H3S10ph (Spearman's Rho $=0.68$ ), and H3K4me2 (Spearman's Rho $=0.92$ ). These data indicate that $\mathrm{X}$ chromosomes in the sexes differ by degree of modification, but the pattern of modifications along the male $\mathrm{X}$ chromosome is shadowed along the female $\mathrm{X}$ chromosome.

Dosage compensation alters transcription relative to autosomes. Therefore, one might expect that genes showing particular modifications in males and females would be expressed. To determine if the three histone marks were associated with transcribed genes, we examined gene expression data for male or female adult flies from a previous study [4] for genes highly enriched or depleted for different histone marks (Figure 5). The genes enriched for any of the three different histone marks all showed significantly higher expression levels compared to genes depleted for histone marks. This observation held in both males and females tissues. For example, the expression level of genes enriched for $\mathrm{H} 4 \mathrm{~K} 16 \mathrm{ac}$ is 2.68-fold higher in males and 3.77-fold higher in females relative to genes depleted for H4K16ac $\left(P<10^{-11}\right.$, Bonferroni corrected KS test) within each sex. These data clearly indicate that the three different histone modifications are correlated inter $s e$ and with expression. We suggest that all three marks contribute to the mild hypertranscription of female $\mathrm{X}$ chromosomes.

Germline X chromosomes are dosage compensated by increased expression of the single $\mathrm{X}$ in males relative to autosomes [4], but germline dosage compensation is not mediated by the MSL complex [33]. Therefore the histone mark and expression correlations may not hold for the germline. To determine if the shadow marks on the female $\mathrm{X}$ chromosome are restricted to genes expressed in the soma, we examined male-biased expression in adult flies, which is dominated by expression within the male germline [2]. If H4K16ac is associated with active

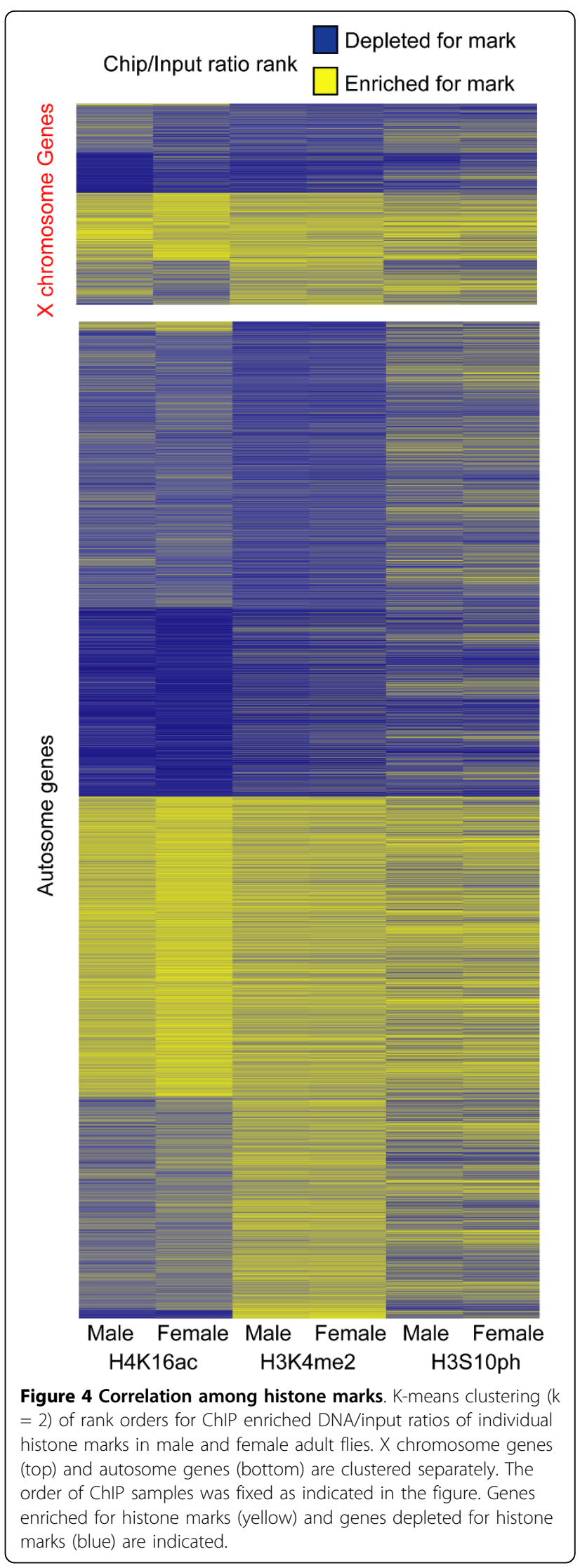




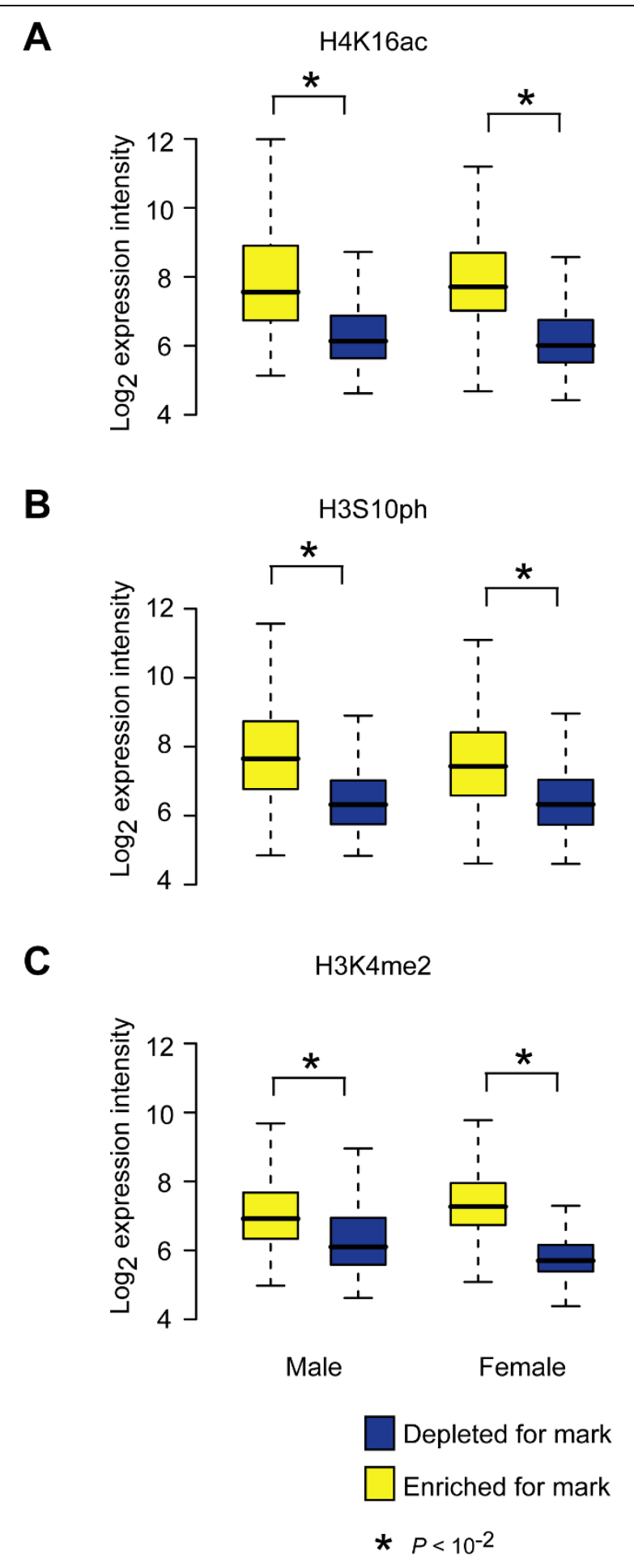

Figure 5 Correlation between marks and expression. Box plots of expression intensities $\left(\log _{2}\right)$ of male or female adult flies for genes enriched for H4K16ac (A), H3S1Oph (B), H3K4me2 (C) (ChIP/ input percentile rank $>90 \%$ ) and genes depleted for those three histone marks (ChIP/input percentile rank $<10 \%$ ) in male or female adult flies. See Figure 1 for box plot format. Significance differences $\left(P<10^{-2}\right.$, Bonferroni corrected KS test) between genes enriched and depleted for histone marks are indicated (asterisks). transcription only in somatic cells as predicted by our understanding of somatic MSL function, then genes with male-biased expression in whole flies should show reduced H4K16ac levels. Indeed, this is what we observed (Figure 6). X chromosome genes showing male-biased expression in whole flies show very low (average ChIP/input ratio $=-0.015)$ H4K16ac levels in males, compared to $\mathrm{X}$ chromosome genes showing nonbiased expression in males (average ChIP/input ratio = 0.368, $P<10^{-12}$, Bonferroni corrected KS test). These data support the idea that dosage compensation marks occur at genes active in the male soma, but not the germline.

Dosage compensation may be not being required for genes that should be differentially regulated in the female and male soma. In support of this idea, we also observed lower H4K16ac among X chromosome genes showing male-biased expression in the soma (average ChIP/input ratio $=0.178$ for genes with male-biased expression vs. 0.351 for genes with non-biased expression, $P<10^{-2}$, Bonferroni corrected KS test). These data are also consistent with the idea that genes with malebiased expression are poorly expressed on the X chromosome due to limited dosage compensation [34]. Interestingly, the same pattern is observed for genes with female-biased expression. We observed lower H4K16ac among genes showing female-biased expression in females (average ChIP/input ratio $=-0.053$ for genes with female-biased expression vs. 0.060 for genes with non-biased expression, $P<10^{-2}$, Bonferroni corrected KS test). These data suggest that sex differentially regulated genes are not H4K16ac regulated.

\section{Discussion}

Our data indicate that the dosage compensation marks associated with $\mathrm{X}$ chromosomes in males are also enriched on $\mathrm{X}$ chromosomes more generally. This is part of a growing body of data showing that the $\mathrm{X}$ chromosome is distinct from the autosomes. For example, previous studies show that Jil1 mutants cause accumulation of the H4K9me2 mark and the transcriptional negative regulator HP1 on the $\mathrm{X}$ chromosome of both males and females [35]. Additionally, 9 of 29 chromatin associated proteins surveyed in female Drosophila Kc167 cells are enriched on the X chromosome [36]. Finally, both Mof and H4K16ac enrichment has been observed on the X chromosomes of female Kc167 cells [24,37], although female results served as controls in those manuscripts and the enrichment in females was not highlighted. Collectively, these data indicate that X chromatin differences from autosomes are not restricted to male-specific dosage compensation. We have previously observed a modest elevation of gene expression from the female $\mathrm{X}$ chromosome relative to autosomes in $D$. 
melanogaster [4] and this same modest (1.11 to 1.24 fold) but highly significant elevation of female $\mathrm{X}$ chromosome expression is consistently observed in six other Drosophila species [9], suggesting that this modest elevation of expression on $\mathrm{X}$ chromosome is independent of microarray platform and species. We suggest that specialized X chromatin contributes to elevated X chromosome gene expression in females in addition to males, albeit to a much more limited extent.

That there is enrichment for $\mathrm{X}$ chromosome marks associated with $\mathrm{X}$ chromosome dosage compensation in males is not de facto evidence of function within females. Indeed, the viability of female mutants for many of the MSL components, indicates that the MSL complex is not required. It is possible that rather than being functional in females, the observed $\mathrm{X}$ chromosome enrichment is a consequence of the evolution of dosage compensation in males $[6,38,39]$. In the sequence of sex chromosome evolution from an autosome pair, genes are gradually lost from the neo-Y resulting in hemizygosity for those genes in males (Figure 7). Any sequence changes that would promote increased $\mathrm{X}$ chromosome expression in males, by for example opening chromatin structure, would be highly advantageous in males. Those sequence changes would also be present in females where they would be disadvantageous. However, if the increase in male fitness does not result in a large negative affect on female fitness, then sequence changes supporting increased epigenetic enhancement of X chromosome expression would be favored. As more genes were lost and the $\mathrm{Y}$ chromosome was reduced to a gene desert, the need for balancing expression with the autosomes became critical, such that the general MSL complex became specialized for the X. MSL is highly deleterious for females, but MSL formation is blocked by the action of Sxl on the MSL component Msl2 [1]. We suggest that Sxl activity is sufficient to ensure female viability, but the cis changes on the $\mathrm{X}$ chromosome and any low level Mof activity in the absence of MSL result in a more open chromatin structure and slight over-expression from the female $\mathrm{X}$ chromosome.

While modest over expression of $\mathrm{X}$ chromosome genes in females may be of little evolutionary consequence, one can envision that more extensive over expression would be detrimental. For example, large duplications are poorly tolerated in Drosophila and [40] trisomy is poorly tolerated in humans [41]. If the $\mathrm{X}$ chromosome tends to be in a favourable state for transcription in both males and females, this could ultimately lead to counter measures in females to prevent functional tetrasomy. This might be what has occurred in mammals, where one $\mathrm{X}$ chromosome is inactivated in females. There is recent evidence that the active $\mathrm{X}$ chromosomes of mammals show increased expression $[4,42,43]$ raising the possibility that $\mathrm{X}$ inactivation is a consequence of $\mathrm{X}$ chromosome dosage compensation in males. The main dosage compensation counter measure in Drosophila females is inhibition of MSL complex formation by Sxl protein [44]. However, if the observed over expression of $\mathrm{X}$ chromosomes in Drosophila females [9] was more extreme, then inactivating an $X$ chromosome in females to counter $\mathrm{X}$ chromosome dosage compensation in males would be logical. This may be how such a mechanism evolved in mammals.

\section{Conclusions}

In this study we examined the general chromatin structure and multiple histone marks in both Drosophila males and females. We found that X chromatin structure is different from autosome structure independent 
Males

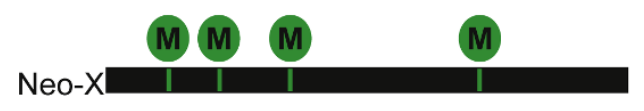

Neo-Y

Cis changes favor open chromatin to increase $\mathrm{X}$ expression

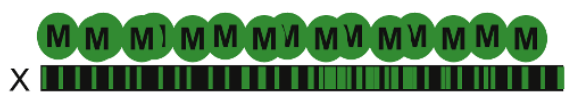

Y

Continued selection (cis and trans)
Females

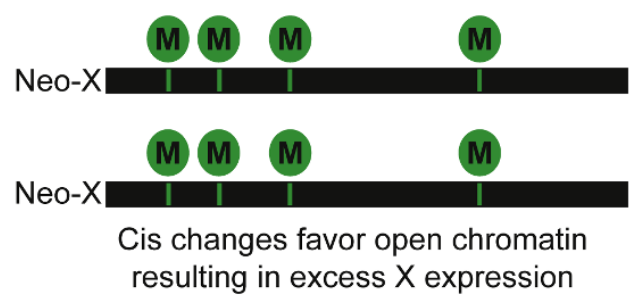

SxI -I D

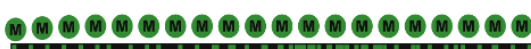

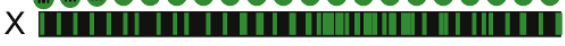

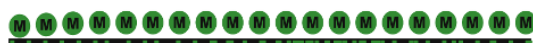

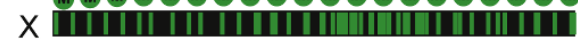

Inhibit trans activator to counter dosage compensation

MSL complex

Figure 7 Model of sex chromosome chromatin structure evolution.

of sex. Histone marks associated with dosage compensation or active transcription that are highly enriched on the male $\mathrm{X}$ chromosome are also slightly enriched on the female $\mathrm{X}$ chromosome. These findings suggest that the distinct female $\mathrm{X}$ chromatin structure is an evolutionary consequence of dosage compensation in males.

\section{Methods}

\section{Chromatin Preparation}

Flies were grown at $25^{\circ} \mathrm{C}$ on standard cornmeal media. Wild type flies $\left(y^{1} w^{67 c}\right)$ for chromatin immunoprecipitation were aged for 5-7 days post-eclosion, sexed and flash frozen. About $0.5 \mathrm{~g}$ of sex-sorted adult flies was cross-linked with $1.8 \%$ formaldehyde in cross-linking solution (50 mM pH 8.0 HEPES buffer, 1 mM EDTA, $0.5 \mathrm{mM}$ EGTA, and $100 \mathrm{mM} \mathrm{NaCl}$ ) for 20 minutes at room temperature on a shaker. We confirmed that this procedure cross-linked the DNA by extracting DNA from treated and untreated flies without reversing the cross-linking and performing gel migration retardation experiments. The cross-linked flies were incubated in PBS supplemented with $125 \mathrm{mM}$ Glycine and 0.01\% Triton for 5 minutes and then washed twice with PBS and $0.01 \%$ Triton. The flies were homogenized and the cells were collected from the pellet after centrifuge at 7500 rpm for 5 minutes at $4^{\circ} \mathrm{C}$. The collected cells from adult flies were disrupted in cell lysis buffer ( $5 \mathrm{mM}$ pH8.0 PIPES buffer, $85 \mathrm{mM}$ Potassium chloride, 0.5\% Nonidet P40 and protease inhibitors) for 10 minutes and then resuspended in nuclei lysis buffer $(50 \mathrm{mM}$ pH8.1 Tris $\mathrm{HCl}, 10 \mathrm{mM}$ EDTA, 1\% SDS and protease inhibitors) for 20 minutes at $4^{\circ} \mathrm{C}$. The nuclear extract was sheared to $200-1000 \mathrm{bp}$ by sonication for 8 minutes on ice (pulsed 8 times for 30 seconds with 30 second intervals) using a Misonix Sonicator 3000 (Misonix, Inc. Farmingdale, NY). After sonication, cell debris was removed by centrifugation and $500 \mathrm{ul}$ of chromatin solution was used for one immunoprecipitation reaction. $5 \mathrm{ul}$ antiH4K16ac (Upstate, 07-329), 5 ul anti-H3S10ph (Upstate, 05-817) or $3 \mathrm{ul}$ anti-H4K3me2 (Upstate, 07-030) was incubated with the chromatin for 2 hours and then was bound to protein A agarose beads at $4^{\circ} \mathrm{C}$ overnight. The beads were washed three times with $0.1 \%$ SDS, $1 \%$ Trition, 2 mM EDTA, $20 \mathrm{mM}$ ph8.0 Tris, $150 \mathrm{mM} \mathrm{NaCl}$; three times with $0.1 \%$ SDS, $1 \%$ Trition, 2 mM EDTA, 20 $\mathrm{mM}$ ph8.0 Tris, $500 \mathrm{mM} \mathrm{NaCl}$; and twice with $10 \mathrm{mM}$ pH8.1 Tris, $1 \mathrm{mM}$ EDTA, $0.25 \mathrm{M} \mathrm{LiCl,} \mathrm{1 \%} \mathrm{NP40,} \mathrm{1 \%}$ sodium deoxycholate. The immunoprecipitated DNA was eluted from the beads in $0.1 \mathrm{M} \mathrm{NaHCO} 3$ and $1 \%$ SDS. Formaldehyde cross-links were reversed by incubation at $65^{\circ} \mathrm{C}$ overnight. DNA was purified by phenolchloroform extraction and ethanol precipitation.

\section{DNA amplification and Array hybridization}

DNA amplification was performed using a Ligationmediated PCR (LM-PCR) protocol [45] from FlyChip http://www.flychip.org.uk/protocols/chip/lm_pcr.php. 600 ng of amplified DNA (ChIP enriched DNA or input DNA) were then labeled using the Cy3- or Cy5-labeled 
random nonamers (Trilink Biosciences, San Diego, USA) with Klenow enzymes. The labeled DNA was purified and resuspended in hybridization buffer as described [46]. For hybridizations we used FlyGEM arrays [46], that are spotted arrays with PCR amplicons biased to the 3' ends of annotated genes, which is a suitable platform given that H4K16ac is found to be present along the whole length of target genes with a 3' bias [47] and given our results on shearing patterns at gene models. Labeling with $\mathrm{Cy} 3$ and $\mathrm{Cy} 5$ random primers was as described $[4,46]$. There is no dye effect using this endlabeling method [46]. Preliminary ChIP-chip experiments on S2 cells showed no dye effects (not shown). All labeling, hybridization and scanning was done under low ozone conditions. We used Cy5 for ChIP and Cy3 for input in all experiments. Four biological replicates (ChIP vs. input) were performed for H4K16ac in males and three biological replicates were performed for H4K16ac in females. Two biological replicates were performed for H3S10ph and H3K4me2 experiments. Arrays were scanned on an Axon GenePix 4000B (Molecular Devices Corporation, Sunnyvale, CA) and signal for each array elements were extracted with GenePix v.5.1 image acquisition software (Molecular Devices Corporation).

\section{DNA-Seq and data handling}

$300 \mathrm{ng}$ of DNA derived from sheared chromatin of female and male adult flies was prepared as outlined for ChIP input controls above. This was used for solexa library preparation using the genomic DNA sample preparation kit (Illumina, San Diego, CA). We size selected libraries ( 200 bp) by excision of the appropriate region following agarose gel electrophoresis. We determined library concentration on a Nanodrop spectrophotometer (NanoDrop products, Wilmington, DE) and hybridized 4 pM of adaptor-ligated DNA to the flow cell. DNA clusters were generated using the Illumina cluster station, followed by 36 cycles of sequencing on the Illumina Genome Analyzer. Image analysis and base calling were performed using a manufacturer-provided computational pipeline (version 0.3) including the Firecrest and Bustard applications and sequence reads were then aligned with the Drosophila melanogaster assembly (BDGP Release 5, dm3 [48]) using Bowtie (v 0.10.0) [49]. We used only uniquely mapped reads with no more than two mismatches.

We calculated average read coverage depth at each base per $\mathrm{Kb}$ per million sequenced base pairs to normalize between samples with slightly different total sequence depth. The average coverage in non-overlapping $1 \mathrm{~kb}$ windows were calculated on each chromosome arm for the distribution of sequence coverage in the genome. We also calculated the average coverage for each annotated [50] exon, intron and $+/-3000 \mathrm{bp}$ around the transcription start sites and transcription termination sites in the genome. To test whether the sequenced reads coverage over non-overlapping $1 \mathrm{~kb}$ windows or different gene features on individual chromosome arms were significantly different from the whole genome, we performed two sample Kolmogorov-Smirnov tests (KS tests) between random sampled coverage values from nonoverlapping $1 \mathrm{~kb}$ windows or gene features on individual chromosome arms and the same number of random sampled coverage values from the whole genome. The coverage for gene features on male $\mathrm{X}$ chromosome was multiplied by 2 to correct for the different number of $\mathrm{X}$ chromosome in male. The sample size for random sampling was 1000 for all chromosome arms except for the $4^{\text {th }}$ chromosome (sample size $=200$ ) and the permutation procedure was repeated 100 times. The distribution of $P$ values from the 100 permutation KS tests were used to determine whether the genes features on one particular chromosome arms were significant different from the gene features in the genome. For visualization purpose, we calculated the average coverage along different gene features in 100 overlapping windows with widow size as $1 / 10$ of the whole region for individual genes and then plotted the moving averaged coverage along different gene features for genes on the $\mathrm{X}$ chromosome and different autosome arms separately.

\section{Microarray data handling}

All microarray data were processed and analyzed in R/ Bioconductor [51] package limma. For the ChIP-chip studies, the $\log _{2}$ ratio between two channels for individual arrays were median-centered and then all the arrays were normalized using quantile normalization based on the input channel across arrays while assuring that the input channel has the same empirical distribution among arrays. Background corrections were then applied to exclude array elements with intensities less than the average intensities of control elements (designed against nonDrosophila DNA) in both channels for downstream analysis. The average intensities of the control elements were also subtracted from the intensities of every array element on two channels separately. Duplicated array elements were merged by calculating the average ratio from duplicated probes. The consistency between biological replicates was checked by the density scatter plot of the input and ChIP DNA intensities among all replicates (see Additional file 1, 2 for H4K16ac ChIP replicates in male adult files, Additional file 3, 4 for H4K16ac ChIP replicates in female adult files and Additional file 5 for other histone marks). The raw and normalized data were inspected to make sure that normalization was appropriate (see Additional file 6).

The comparisons of the distribution of ChIP/input ratios or ChIP input intensities among different chromosome arms were performed using two sample 
Kolmogorov-Smirnov tests (KS tests) and the $P$ values were adjusted using the Bonferroni correction for multiple-comparison. The maximum adjusted $P$ value was truncated at 1.0. KS tests and the Bonferroni corrections were applied to ChIP/input ratios from individual replicates and averaged ratios to test the significance of $\mathrm{X}$ chromosome in different replicates. Unless otherwise noted, all ChIP/input ratios used in the figures were averaged across all the biological replicates. Spearman's Rank Correlation was used to test the relationship between H4K16ac level and all other histone modifications in males and females. To test the correlation among different histone marks, we also used the ChIP/ input ratio ranks in each ChIP sample for K-means clustering analysis with 2 nodes using the Euclidean similarity metric. X chromosome genes and autosome genes were clustered separately and the order of ChIP samples was fixed as indicated in the figure in both clustering analysis. Data were clustered and visualized using Cluster3.0/Tree-View. The expression data for sex-sorted whole adults and for gonadectomized male and female carcasses were from GEO accession number GSE6640 and GSE442. The expression values used for the correlation between marks and expression were averaged expression values from all replicates in each dataset. For the correlation between expression and different histone marks, genes with ChIP/input ratio percentile rank $>90 \%$ were used as genes with enriched marks and genes with ChIP/input ratio percentile rank $<10 \%$ were used as genes with depleted marks to assure similar sample size for different histone marks. The list of genes with male-, female- or non-biased expression in adult flies were got from a previous study [52] and the list of genes with sex-biased expression in gonadectomized carcasses were produced from the data set GSE442 using the same analysis approach [52].

\section{Data sources}

All DNA-Seq and ChIP-chip data and full platform description are available at GEO under accession number GSE15593.

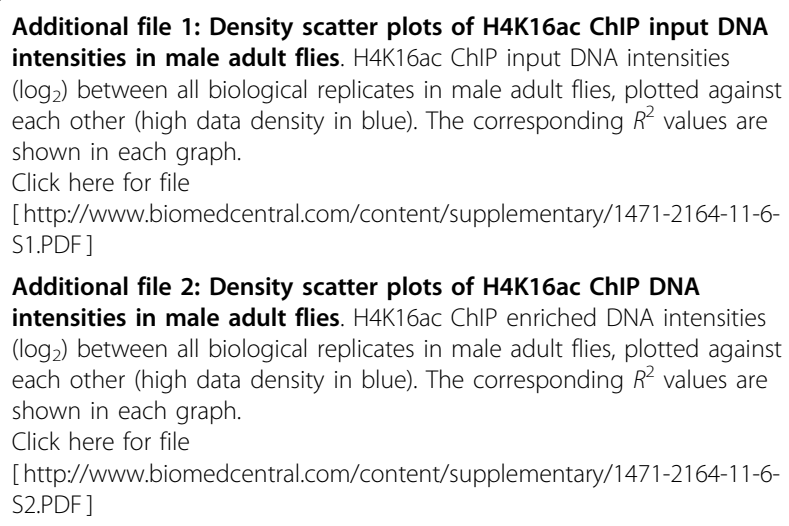

Additional file 3: Density scatter plots of H4K16ac ChIP input DNA intensities in female adult flies. H4K16ac ChIP input DNA intensities $\left(\log _{2}\right)$ between all biological replicates in female adult flies, plotted against each other (high data density in blue). The corresponding $R^{2}$ values are shown in each graph.

Click here for file

[ http://www.biomedcentral.com/content/supplementary/1471-2164-11-6S3.PDF ]

Additional file 4: Density scatter plots of H4K16ac ChIP DNA intensities in female adult flies. H4K16ac ChIP enriched DNA intensities $\left(\log _{2}\right)$ between all biological replicates in female adult flies, plotted against each other (high data density in blue). The corresponding $R^{2}$ values are shown in each graph.

Click here for file

[ http://www.biomedcentral.com/content/supplementary/1471-2164-11-6S4.PDF ]

Additional file 5: Density scatter plots of H3K4me2 and H3S1Oph ChIP-chip intensities in male and female adult flies. H3K4me2 and H3S10ph ChIP input (A) or enriched DNA (B) intensities $\left(\log _{2}\right)$ between biological replicates, plotted against each other (high data density in blue). The corresponding $R^{2}$ values are shown in each graph.

Click here for file

[http://www.biomedcentral.com/content/supplementary/1471-2164-11-6S5.PDF ]

Additional file 6: Density MA plots of normalized ChIP experiments. ChIP/input ratio $\left(\log _{2}\right)$ versus average ChIP and input intensity $\left(\log _{2}\right)$ plots for H4K16ac ChIP (A-B), H3S10ph ChIP (C-D) and H3K4me2 ChIP (EF) in male and female adult flies (high data density in blue). Each MA plot represents data from one biological replicate of each ChIP data set. Click here for file

[http://www.biomedcentral.com/content/supplementary/1471-2164-11-6S6.PDF ]

\section{Acknowledgements}

We thank the NIDDK genomics core laboratory for solexa sequencing. We also thank Shirley Liu, David Sturgill, and John Malone for discussions and preliminary analysis of DNA-Seq data. The authors are grateful to members of Oliver lab, Brian Charlesworth and the dosage compensation community for many helpful comments. This research was supported by the Intramural Research Program of the NIH, NIDDK.

\section{Authors' contributions}

YZ designed the project, collected and analyzed the data and wrote the manuscript. $\mathrm{BO}$ designed the experiments, provided guidance in interpreting the results and wrote the manuscript. Both authors read and approved the final manuscript.

Received: 15 April 2009

Accepted: 5 January 2010 Published: 5 January 2010

\section{References}

1. Lucchesi JC, Kelly WG, Panning B: Chromatin remodeling in dosage compensation. Annu Rev Genet 2005, 39:615-651.

2. Vicoso $B$, Charlesworth B: Evolution on the $X$ chromosome: unusual patterns and processes. Nat Rev Genet 2006, 7:645-653.

3. Muller HJ, Kaplan WD: The dosage compensation of Drosophila and mammals as showing the accuracy of the normal type. Genet Res 1966, 8:41-59.

4. Gupta V, Parisi M, Sturgill D, Nuttall R, Doctolero M, Dudko OK, Malley JD, Eastman PS, Oliver B: Global analysis of X-chromosome dosage compensation. J Biol 2006, 5:3.

5. Lucchesi JC: Dosage compensation in Drosophila. Isozymes Curr Top Biol Med Res 1983, 9:179-188.

6. Charlesworth B: The evolution of chromosomal sex determination and dosage compensation. Curr Biol 1996, 6:149-162.

7. Lande R: Sexual Dimorphism, Sexual Selection, and Adaptation in Polygenic Characters. Evolution 1980, 34:292-305. 
8. Bhadra U, Pal-Bhadra M, Birchler JA: Role of the male specific lethal (msl) genes in modifying the effects of sex chromosomal dosage in Drosophila. Genetics 1999, 152:249-268.

9. Sturgill $D$, Zhang $Y$, Parisi $M$, Oliver B: Demasculinization of $X$ chromosomes in the Drosophila genus. Nature 2007, 450:238-241.

10. Straub T, Becker PB: Dosage compensation: the beginning and end of generalization. Nat Rev Genet 2007, 8:47-57.

11. Zhang Y, Oliver B: Dosage compensation goes global. Curr Opin Genet Dev 2007, 17:113-120.

12. Gross DS, Garrard WT: Nuclease hypersensitive sites in chromatin. Annu Rev Biochem 1988, 57:159-197.

13. Teytelman L, Ozaydin B, Zill O, Lefrancois P, Snyder M, Rine J, Eisen MB: Impact of chromatin structures on DNA processing for genomic analyses. PLoS One 2009, 4:e6700.

14. Auerbach RK, Euskirchen G, Rozowsky J, Lamarre-Vincent N, Moqtaderi Z, Lefrancois P, Struhl K, Gerstein M, Snyder M: Mapping accessible chromatin regions using Sono-Seq. Proc Natl Acad Sci USA 2009, 106:14926-14931.

15. Schwartz YB, Kahn TG, Pirrotta V: Characteristic low density and shear sensitivity of cross-linked chromatin containing polycomb complexes. Mol Cell Biol 2005, 25:432-439.

16. Ruthenburg AJ, Li H, Patel DJ, Allis CD: Multivalent engagement of chromatin modifications by linked binding modules. Nat Rev Mol Cell Biol 2007, 8:983-994.

17. Jenuwein T, Allis CD: Translating the histone code. Science 2001, 293:10741080.

18. Gupta A, Guerin-Peyrou TG, Sharma GG, Park C, Agarwal M, Ganju RK, Pandita S, Choi K, Sukumar S, Pandita RK, Ludwig T, Pandita TK: The mammalian ortholog of Drosophila MOF that acetylates histone $\mathrm{H} 4$ lysine 16 is essential for embryogenesis and oncogenesis. Mol Cell Biol 2008, 28:397-409.

19. Taipale M, Rea S, Richter K, Vilar A, Lichter P, Imhof A, Akhtar A: hMOF histone acetyltransferase is required for histone $\mathrm{H} 4$ lysine 16 acetylation in mammalian cells. Mol Cell Biol 2005, 25:6798-6810.

20. Smith ER, Eisen A, Gu W, Sattah M, Pannuti A, Zhou J, Cook RG, Lucchesi JC, Allis CD: ESA1 is a histone acetyltransferase that is essential for growth in yeast. Proc Natl Acad Sci USA 1998, 95:3561-3565.

21. Hilfiker A, Hilfiker-Kleiner D, Pannuti A, Lucchesi JC: mof, a putative acetyl transferase gene related to the Tip60 and MOZ human genes and to the SAS genes of yeast, is required for dosage compensation in Drosophila. Embo J 1997, 16:2054-2060.

22. Lavender JS, Birley AJ, Palmer MJ, Kuroda MI, Turner BM: Histone H4 acetylated at lysine 16 and proteins of the Drosophila dosage compensation pathway co-localize on the male $\mathrm{X}$ chromosome through mitosis. Chromosome Res 1994, 2:398-404.

23. Bone JR, Lavender J, Richman R, Palmer MJ, Turner BM, Kuroda MI: Acetylated histone $\mathrm{H} 4$ on the male $\mathrm{X}$ chromosome is associated with dosage compensation in Drosophila. Genes Dev 1994, 8:96-104.

24. Kind J, Vaquerizas JM, Gebhardt P, Gentzel M, Luscombe NM, Bertone P, Akhtar A: Genome-wide analysis reveals MOF as a key regulator of dosage compensation and gene expression in Drosophila. Cell 2008, 133:813-828

25. Birchler JA, Fernandez HR, Kavi HH: Commonalities in compensation. Bioessays 2006, 28:565-568.

26. Bao X, Cai W, Deng H, Zhang W, Krencik R, Girton J, Johansen J, Johansen KM: The $\mathrm{COOH}$-terminal domain of the JIL-1 histone H3S10 kinase interacts with histone $\mathrm{H} 3$ and is required for correct targeting to chromatin. J Biol Chem 2008, 283:32741-32750.

27. Jin $Y$, Wang $Y$, Johansen J, Johansen KM: JIL-1, a chromosomal kinase implicated in regulation of chromatin structure, associates with the male specific lethal (MSL) dosage compensation complex. J Cell Biol 2000, 149:1005-1010.

28. Jin Y, Wang Y, Walker DL, Dong H, Conley C, Johansen J, Johansen KM: JIL1: a novel chromosomal tandem kinase implicated in transcriptional regulation in Drosophila. Mol Cell 1999, 4:129-135.

29. Lerach S, Zhang W, Deng H, Bao X, Girton J, Johansen J, Johansen KM: JIL-1 kinase, a member of the male-specific lethal (MSL) complex, is necessary for proper dosage compensation of eye pigmentation in Drosophila. Genesis 2005, 43:213-215.

30. Barski A, Zhao K: Genomic location analysis by ChIP-Seq. J Cell Biochem 2009, 107:11-18.
31. Riddle NC, Shaffer CD, Elgin SC: A lot about a little dot - lessons learned from Drosophila melanogaster chromosome 4. Biochem Cell Biol 2009, 87:229-241.

32. Sabo PJ, Humbert R, Hawrylycz M, Wallace JC, Dorschner MO, MCArthur M, Stamatoyannopoulos JA: Genome-wide identification of DNasel hypersensitive sites using active chromatin sequence libraries. Proc Nat Acad Sci USA 2004, 101:4537-4542.

33. Rastelli L, Kuroda Ml: An analysis of maleless and histone $\mathrm{H} 4$ acetylation in Drosophila melanogaster spermatogenesis. Mech Dev 1998, 71:107-117.

34. Vicoso $B$, Charlesworth $B$ : The deficit of male-biased genes on the $D$. melanogaster $\mathrm{X}$ chromosome is expression-dependent: a consequence of dosage compensation?. J Mol Evol 2009, 68:576-583.

35. Zhang W, Deng H, Bao X, Lerach S, Girton J, Johansen J, Johansen KM: The JIL-1 histone H3S10 kinase regulates dimethyl H3K9 modifications and heterochromatic spreading in Drosophila. Development 2006, 133:229-235.

36. de Wit E, Braunschweig U, Greil F, Bussemaker HJ, van Steensel B: Global chromatin domain organization of the Drosophila genome. PLoS Genet 2008, 4:e1000045.

37. Gelbart ME, Larschan E, Peng S, Park PJ, Kuroda MI: Drosophila MSL complex globally acetylates $\mathrm{H} 4 \mathrm{~K} 16$ on the male $\mathrm{X}$ chromosome for dosage compensation. Nat Struct Mol Biol 2009, 16:825-832.

38. Ohno S: Sex chromosomes and sex-linked genes Berlin, New York;: Springer 1967.

39. Charlesworth B: Model for evolution of $Y$ chromosomes and dosage compensation. Proc Natl Acad Sci USA 1978, 75:5618-5622.

40. Lindsley DL, Sandler L, Baker BS, Carpenter AT, Denell RE, Hall JC, Jacobs PA, Miklos GL, Davis BK, Gethmann RC, Hardy RW, Steven AH, Miller M, Nozawa H, Parry DM, Gould-Somero M, Gould-Somero M: Segmental aneuploidy and the genetic gross structure of the Drosophila genome. Genetics 1972, 71:157-184.

41. Payer B, Lee JT: $\mathbf{X}$ chromosome dosage compensation: how mammals keep the balance. Annu Rev Genet 2008, 42:733-772.

42. Lin H, Gupta V, Vermilyea MD, Falciani F, Lee JT, O'Neill LP, Turner BM: Dosage compensation in the mouse balances up-regulation and silencing of X-linked genes. PLoS Biol 2007, 5:e326.

43. Nguyen DK, Disteche CM: Dosage compensation of the active $X$ chromosome in mammals. Nat Genet 2006, 38:47-53.

44. Gilfillan GD, Dahlsveen IK, Becker PB: Lifting a chromosome: dosage compensation in Drosophila melanogaster. FEBS Lett 2004, 567:8-14.

45. Birch-Machin I, Gao S, Huen D, McGirr R, White RA, Russell S: Genomic analysis of heat-shock factor targets in Drosophila. Genome Biol 2005, 6: R63.

46. Johnston R, Wang B, Nuttall R, Doctolero M, Edwards P, Lu J, Vainer M, Yue H, Wang X, Minor J, Chan C, Lash A, Goralski T, Parisi M, Oliver B, Eastman S: FlyGEM, a full transcriptome array platform for the Drosophila community. Genome Biol 2004, 5:R19.

47. Smith $E R$, Allis CD, Lucchesi JC: Linking global histone acetylation to the transcription enhancement of X-chromosomal genes in Drosophila males. J Biol Chem 2001, 276:31483-31486.

48. Adams MD, Celniker SE, Holt RA, Evans CA, Gocayne JD, Amanatides PG, Scherer SE, Li PW, Hoskins RA, Galle RF, George RA, Lewis SE, Richards S, Ashburner M, Henderson SN, Sutton GG, Wortman JR, Yandell MD, Zhang Q, Chen LX, Brandon RC, Rogers YH, Blazej RG, Champe M, Pfeiffer BD, Wan KH, Doyle C, Baxter EG, Helt G, Nelson CR, Gabor GL, Abril JF, Agbayani A, An HJ, Andrews-Pfannkoch C, Baldwin D, Ballew RM, Basu A, Baxendale J, Bayraktaroglu L, Beasley EM, Beeson KY, Benos PV, Berman BP, Bhandari D, Bolshakov S, Borkova D, Botchan MR, Bouck J, Brokstein P, Brottier P, Burtis KC, Busam DA, Butler H, Cadieu E, Center A, Chandra I, Cherry JM, Cawley S, Dahlke C, Davenport LB, Davies P, de Pablos B, Delcher A, Deng Z, Mays AD, Dew I, Dietz SM, Dodson K, Doup LE, Downes M, Dugan-Rocha S, Dunkov BC, Dunn P, Durbin KJ, Evangelista CC, Ferraz C, Ferriera S, Fleischmann W, Fosler C, Gabrielian AE, Garg NS, Gelbart WM, Glasser K, Glodek A, Gong F, Gorrell JH, Gu Z, Guan P, Harris M, Harris NL, Harvey D, Heiman TJ, Hernandez JR, Houck J, Hostin D, Houston KA, Howland TJ, Wei MH, Ibegwam C, Jalali M, Kalush F, Karpen GH, Ke Z, Kennison JA, Ketchum KA, Kimmel BE, Kodira CD, Kraft C, Kravitz S, Kulp D, Lai Z, Lasko P, Lei Y, Levitsky AA, Li J, Li Z, Liang Y, Lin X, Liu X, Mattei B, Mclntosh TC, McLeod MP, McPherson D, Merkulov G, Milshina NV, Mobarry C, Morris J, Moshrefi A, Mount SM, Moy M, Murphy B, Murphy L, Muzny DM, Nelson DL, Nelson DR, Nelson KA, Nixon K, Nusskern DR, Pacleb JM, Palazzolo M, Pittman GS, Pan S, Pollard J, Puri V, 
Reese MG, Reinert K, Remington K, Saunders RD, Scheeler F, Shen H, Shue BC, Siden-Kiamos I, Simpson M, Skupski MP, Smith T, Spier E,

Spradling AC, Stapleton M, Strong R, Sun E, Svirskas R, Tector C, Turner R, Venter $E$, Wang AH, Wang $X$, Wang ZY, Wassarman DA, Weinstock GM, Weissenbach J, Williams SM, Woodage T, Worley KC, Wu D, Yang S, Yao QA, Ye J, Yeh RF, Zaveri JS, Zhan M, Zhang G, Zhao Q, Zheng L, Zheng XH, Zhong FN, Zhong W, Zhou X, Zhu S, Zhu X, Smith HO, Gibbs RA, Myers EW, Rubin GM, Venter JC: The genome sequence of Drosophila melanogaster. Science 2000, 287:2185-2195.

49. Langmead B, Trapnell C, Pop M, Salzberg SL: Ultrafast and memoryefficient alignment of short DNA sequences to the human genome. Genome Biol 2009, 10:R25.

50. Drysdale R: FlyBase: a database for the Drosophila research community. Methods Mol Biol 2008, 420:45-59.

51. Gentleman RC, Carey VJ, Bates DM, Bolstad B, Dettling M, Dudoit S, Ellis B, Gautier L, Ge Y, Gentry J, Hornik K, Hothorn T, Huber W, lacus S, rrizarry R, Leisch F, Li C, Maechler M, Rossini AJ, Sawitzki G, Smith C, Smyth G,

Tierney L, Yang JY, Zhang J: Bioconductor: open software development for computational biology and bioinformatics. Genome Biol 2004, 5:R80.

52. Zhang Y, Sturgill D, Parisi M, Kumar S, Oliver B: Constraint and turnover in sex-biased gene expression in the genus Drosophila. Nature 2007, 450:233-237.

doi:10.1186/1471-2164-11-6

Cite this article as: Zhang and Oliver: An evolutionary consequence of dosage compensation on Drosophila melanogaster female X-chromatin structure?. BMC Genomics 2010 11:6.

Publish with BioMed Central and every scientist can read your work free of charge

"BioMed Central will be the most significant development for disseminating the results of biomedical research in our lifetime. "

Sir Paul Nurse, Cancer Research UK

Your research papers will be:

- available free of charge to the entire biomedical community

- peer reviewed and published immediately upon acceptance

- cited in PubMed and archived on PubMed Central

- yours - you keep the copyright 